\title{
EQUIVALENCE PROBLEMS \\ IN PROJECTIVE DIFFERENTIAL GEOMETRY
}

BY

\author{
KICHOON YANG
}

\begin{abstract}
ABSTRACr. Equivalence problems for abstract, and induced, projective structures are investigated. (i) The notion of induced projective structures on submanifolds of a projective space is rigorously defined. (ii) Equivalence problems for such structures are discussed; in particular, it is shown that nonplanar surfaces in $\mathbf{R} P^{3}$ are all projectively equivalent to each other. (iii) The imbedding problem of abstract projective structures is studied; in particular, we show that every abstract projective structure on a 2-manifold arises as an induced structure on an arbitrary nonplanar surface in $\mathbf{R} P^{3}$; this result should be contrasted to that of Chern (see [6]).
\end{abstract}

Introduction. In [6] Chern showed that any projective connection (a Cartan connection) on an $n$-manifold can be imbedded in $\mathbf{R} P^{N}$, with $N=n(n+1) / 2+$ $[n / 2]$, in that the given projective connection is realized as a restriction of the Maurer-Cartan form of $\operatorname{PGL}(N+1 ; \mathbf{R})$, the group of projective transformations. Consequently, any (abstract) projective structure on an $n$-manifold arises as an induced projective structure on a submanifold of $\mathbf{R} P^{N}$. We observe that the dimension $N$ is larger than the one obtained for Riemannian geometry by Schlafli, Cartan and Janet.

Our purpose is to lay a rigorous foundation for the theory of induced projective structures and (extrinsic) equivalence problems arising from it. In particular, we show that the minimal dimensions required to imbed abstract projective structures are considerably smaller than those required to imbed projective connections.

This paper is organized as follows:

$\$ 1$ defines the notions of abstract projective structures and their equivalences and introduces local expressions and notation.

$\$ 2$ contains a brief exposition on constructing higher order moving frames. In particular, we give a characterization of second order type surfaces in $\mathbf{R} P^{3}$ (Theorem 2.11).

In $\S \S 3$ and 4 we give a rigorous definition of induced projective structures and prove some theorems on extrinsic equivalences. In particular, we show that generic hypersurfaces in $\mathbf{R} P^{n}$ are all extrinsically equivalent to each other (in the sense of Theorem 4.14) if and only if $n \leqslant 3$.

In $\S 5$ we study the problem of imbedding a given abstract projective structure. We prove, in particular, that any abstract structure on a 2-manifold can be imbedded into $\mathbf{R} P^{3}$ (Theorem 5.2).

Received by the editors October 22, 1982 and, in revised form, February 24, 1983.

1980 Mathematics Subject Classification. Primary 53B10.

(C)1984 American Mathematical Society $0002-9947 / 84 \$ 1.00+\$ .25$ per page 
We shall work within the category of smooth maps and objects, except in $\S \S 4$ and 5, where we apply Cartan-Kähler theory, in which case we must assume real analyticity. (Such assumptions will be explicitly made.)

The following index convention will be used throughout:

$$
\begin{aligned}
1 & \leqslant \alpha, \beta, \gamma, \ldots \leqslant n ; \\
1 & \leqslant i, j, k, \ldots \leqslant p ; \\
p+1 & \leqslant a, b, c, \ldots \leqslant n .
\end{aligned}
$$

The author would like to express deep gratitude to Gary Jensen, under whose direction this paper was prepared, for his constant support during the preparation of this paper.

1. Abstract projective structures. In this section, following the expositions of Ehresmann, Kobayashi and Nagano, we explain the notions of abstract projective structures and their equivalences (see [11]). Though there are other approaches (e.g., covariant differentiations in projective tangent bundles), this one provides a unifying language which enables us to consider equivalence problems in general.

$\mathbf{R} P^{n}$ is the real projective space of dimension $n$ which we realize as $\mathbf{R}^{n+1^{*}} / \mathbf{R}^{*}$, where $\mathbf{R}^{n+1^{*}}$ is the set of all nonzero vectors in $\mathbf{R}^{n+1}$ and $\mathbf{R}^{*}$ is the group of nonzero real numbers. For a vector in $\mathbf{R}^{n+1^{*}}$, we denote its equivalence class in $\mathbf{R} P^{n}$ by [ ]. Let $\xi^{0}, \xi^{1}, \ldots, \xi^{n}$ be the homogeneous coordinate system in $\mathbf{R} P^{n}$. Then $x^{\alpha}=\xi^{\alpha} / \xi^{0}$, $\alpha=1,2, \ldots, n$, defines the inhomogeneous coordinate system around ${ }^{\mathrm{t}}[1,0, \ldots, 0]$ which we call the origin of $\mathbf{R} P^{n}$.

$\operatorname{PGL}(n+1 ; \mathbf{R})$, the group of projective transformations, is $\mathrm{GL}(n+1 ; \mathbf{R})$ modulo its center. Once again we use [ ] to denote the equivalence classes. Let $\mathfrak{O K}_{0}$ be $\mathbf{R}^{n}$, and $\mathscr{N}_{0}^{*}$ its dual; so an element of $\mathfrak{M}_{0}$ will be a column vector and an element of $\mathfrak{K}_{0}^{*}$ a row vector. Then the Lie algebra $\mathfrak{p g l}(n+1 ; \mathbf{R})$ of $\operatorname{PGL}(n+1 ; \mathbf{R})$ is the vector space direct sum $\mathfrak{p g l}(n+1 ; \mathbf{R})=\mathfrak{N}_{0} \oplus \mathfrak{g} \mathfrak{l}(n ; \mathbf{R}) \oplus \mathfrak{N}_{0}^{*}$ with the bracket operation:

$$
\begin{aligned}
{[w, z] } & =\left[w^{*}, z^{*}\right]=0, \quad\left[w^{*}, W\right]=w^{*} W, \quad[W, w]=W w, \\
{[W, Z] } & =W Z-Z W, \quad\left[w, w^{*}\right]=w w^{*}+w^{*} w I_{n},
\end{aligned}
$$

where $w, z \in \mathfrak{M}_{0}, w^{*}, z^{*} \in \mathfrak{M}_{0}^{*}$, and $W, Z \in \mathfrak{g l}(n ; \mathbf{R})$. If

$$
\left(X_{B}^{A}\right)_{0 \leqslant A, B \leqslant n} \in \mathrm{GL}(n+1 ; \mathbf{R})
$$

with $X_{0}^{0} \neq 0$, then set $a^{\alpha}=X_{0}^{\alpha} / X_{0}^{0}, a_{\beta}^{\alpha}=x_{\beta}^{\alpha} / x_{0}^{0}$, and $a_{\beta}=x_{\beta}^{0} / x_{0}^{0}$, where $1 \leqslant \alpha$, $\beta \leqslant n$. We shall take $\left(a^{\alpha}, a_{\beta}^{\alpha}, a_{\beta}\right)$ as a local coordinate system in the neighborhood of the identity of $\operatorname{PGL}(n+1 ; \mathbf{R})$ defined by $x_{0}^{0} \neq 0$. Let $\left(\Omega^{\alpha}, \Omega_{\beta}^{\alpha}, \Omega_{\beta}\right)$ be the Maurer-Cartan form of $\operatorname{PGL}(n+1 ; \mathbf{R})$. It is $\mathscr{N}_{0} \oplus \mathfrak{g l}(n ; \mathbf{R}) \oplus \mathscr{N}_{0}^{*}$-valued and components are the left invariant 1 -forms on $\operatorname{PGL}(n+1 ; \mathbf{R})$ which coincide with $d a^{\alpha}, d a_{\beta}^{\alpha}, d a_{\beta}$ at the identity. We record the structure equations:

$$
\begin{aligned}
& d \Omega^{\alpha}=-\Omega_{\gamma}^{\alpha} \wedge \Omega^{\gamma}, \\
& d \Omega_{\beta}^{\alpha}=-\Omega_{\gamma}^{\alpha} \wedge \Omega_{\beta}^{\gamma}-\Omega^{\alpha} \wedge \Omega_{\beta}+\delta_{\beta}^{\alpha} \Omega_{\gamma} \wedge \Omega^{\gamma}, \\
& d \Omega_{\beta}=-\Omega_{\gamma} \wedge \Omega_{\beta}^{\gamma} .
\end{aligned}
$$

The standard action of $\operatorname{GL}(n+1 ; \mathbf{R})$ on $\mathbf{R}^{n+1}$ induces the action of $\operatorname{PGL}(n+1 ; \mathbf{R})$ on $\mathbf{R} P^{n}$. If $\left(a^{\alpha}, a_{\beta}^{\alpha}, a_{\beta}\right) \in \operatorname{PGL}(n+1 ; \mathbf{R})$, then the action is given, in terms of the 
inhomogeneous coordinate system, by the following linear fractional transformation:

$$
\left(x^{\alpha}\right) \mapsto\left(a^{\alpha}+a_{\gamma}^{\alpha} x^{\gamma}\right) /\left(1+a_{\gamma} x^{\gamma}\right) .
$$

The action is transitive and the isotropy subgroup at the origin is

$$
G_{0}=\left\{\left[\begin{array}{cc}
1 & \left(a_{\beta}\right) \\
0 & \\
\cdot & \\
\cdot & \left(a_{\beta}^{\alpha}\right) \\
0 &
\end{array}\right]:\left(a_{\beta}^{\alpha}\right) \in \mathrm{GL}(n ; \mathbf{R}),{ }^{\mathrm{t}}\left(a_{\beta}\right) \in \mathbf{R}^{n}\right\},
$$

i.e., $\left(a^{\alpha}\right) \equiv 0$. If we let $\hat{a}_{\beta}^{\alpha}=a_{\beta}^{\alpha}-a^{\alpha} a_{\beta}$, then (1.2) can be written as

$$
\left(x^{\alpha}\right) \mapsto a^{\alpha}+\hat{a}_{\beta}^{\alpha} x^{\beta}-\frac{1}{2}\left(\hat{a}_{\beta}^{\alpha} a_{\gamma}+\hat{a}_{\gamma}^{\alpha} a_{\beta}\right) x^{\beta} x^{\gamma}+\text { higher order terms. }
$$

Let $Q\left(\mathbf{R} P^{n}\right)$ denote the principal bundle of quadratic frames over $\mathbf{R} P^{n}$ (i.e., the bundle of 2-jets of local diffeomorphisms $\left.\left(\mathbf{R}^{n}, 0\right) \rightarrow \mathbf{R} P^{n}\right)$. Let $\left(\tilde{a}^{\alpha}, \tilde{a}_{\beta}^{\alpha}, \tilde{a}_{\beta \gamma}^{\alpha}\right)$ be the natural local coordinate system in $Q\left(\mathbf{R} P^{n}\right)$ arising from the inhomogeneous coordinate system in $\mathbf{R} P^{n}$. Restricting to $\left(\tilde{a}^{\alpha}\right) \equiv 0$, we obtain the coordinate system on the structure group (denoted $G^{2}(n)$ ), which we write as $\left(\tilde{b}_{\beta}^{\alpha}, \tilde{b}_{\beta \gamma}^{\alpha}\right)$. The right action of $G^{2}(n)$ is given by

$$
\left(\tilde{a}^{\alpha}, \tilde{a}_{\beta}^{\alpha}, \tilde{a}_{\beta \gamma}^{\alpha}\right)\left(\tilde{b}_{\beta}^{\alpha}, \tilde{b}_{\beta \gamma}^{\alpha}\right)=\left(\tilde{a}^{\alpha}, \tilde{a}_{\delta}^{\alpha} \tilde{b}_{\beta}^{\delta}, \tilde{a}_{\delta}^{\alpha} \tilde{b}_{\beta \gamma}^{\delta}+\tilde{a}_{\delta \varepsilon}^{\alpha} \tilde{b}_{\beta}^{\delta} \tilde{b}_{\gamma}^{\varepsilon}\right)
$$

Similarly, we introduce a coordinate system $\left(\tilde{a}^{\alpha}, \tilde{a}_{\beta}^{\alpha}\right)$ in $L\left(\mathbf{R} P^{n}\right)$, the bundle of linear frames, so that the natural projection $Q\left(\mathbf{R} P^{n}\right) \rightarrow L\left(\mathbf{R} P^{n}\right)$ is given by

$$
\left(\tilde{a}^{\alpha}, \tilde{a}_{\beta}^{\alpha}, \tilde{a}_{\beta \gamma}^{\alpha}\right) \rightarrow\left(\tilde{a}^{\alpha}, \tilde{a}_{\beta}^{\alpha}\right) .
$$

If we let $E_{\alpha}$ and $E_{\alpha}^{\beta}$ be the basis for a $(n ; \mathbf{R}) \simeq \mathbf{R}^{n} \oplus \mathrm{gl}(n ; \mathbf{R})$, given by $E_{\alpha}=\partial / \partial \tilde{a}^{\alpha}$ and $E_{\alpha}^{\beta}=\partial / \partial \tilde{a}_{\beta}^{\alpha}$, then the cannonical form of $Q\left(\mathbf{R} P^{n}\right), \Theta$, has the local expression

$$
\Theta=\theta^{\alpha} \otimes E_{\alpha} \oplus \theta_{\beta}^{\alpha} \otimes E_{\alpha}^{\beta}=\left(\theta^{\alpha}, \theta_{\beta}^{\alpha}\right),
$$

where

$$
\begin{gathered}
\theta^{\alpha}=\left(\tilde{a}^{-1}\right)_{\gamma}^{\alpha} d \tilde{a}^{\gamma}, \\
\theta_{\beta}^{\alpha}=\left(\tilde{a}^{-1}\right)_{\gamma}^{\alpha} d \tilde{a}_{\beta}^{\gamma}-\left(\tilde{a}^{-1}\right)_{\gamma}^{\alpha} \tilde{a}_{\delta \beta}^{\gamma}\left(\tilde{a}^{-1}\right)_{\varepsilon}^{\delta} d \tilde{a}^{\varepsilon} .
\end{gathered}
$$

It follows that $d \theta^{\alpha}=-\theta_{\gamma}^{\alpha} \wedge \theta^{\gamma}$.

Observe that the above description is general in that one can replace $\mathbf{R} P^{n}$ and the inhomogeneous coordinate system by an $n$-dimensional manifold $M$ and a local coordinate system in it. For details of the properties of $Q(M)$ and $\Theta$, we refer to [10, Chapter VI, §5].

We now recall (1.3) and write the local expression for the obvious bundle imbedding $I: \operatorname{PGL}(n+1 ; \mathbf{R}) \rightarrow Q\left(\mathbf{R} P^{n}\right):$

$$
\left(a^{\alpha}, a_{\beta}^{\alpha}, a_{\beta}\right) \mapsto\left(a^{\alpha}, \hat{a}_{\beta}^{\alpha},-\left(\hat{a}_{\beta}^{\alpha} a_{\gamma}+\hat{a}_{\gamma}^{\alpha} a_{\beta}\right)\right) .
$$

Note that at the structure group level $\hat{a}_{\beta}^{\alpha}=a_{\beta}^{\alpha}$, and restricting to $\left(a^{\alpha}\right) \equiv 0$, we obtain a faithful representation of $G_{0}$ into $G^{2}(n)$. From now on we use $\left(a^{\alpha}, \hat{a}_{\beta}^{\alpha}, a_{\beta}\right)$ as the standard coordinates on $\operatorname{PGL}(n+1 ; \mathbf{R})$, and by abusing notation, we drop the . 
We remark that the crucial difference between Riemannian geometry and projective geometry is due to the fact that the Euclidian group $\mathbf{E}(n)$ is naturally a subbundle of $L\left(\mathbf{R}^{n}\right)$, whereas $\operatorname{PGL}(n+1 ; \mathbf{R})$ is a subbundle of $Q\left(\mathbf{R} P^{n}\right)$.

Let $M$ be a manifold of dimension $n$. Then an (abstract) projective structure $P$ over $M$ is defined to be a $G_{0}$-principal subbundle of $Q(M)$ with the group imbedding coming from (1.4).

Definition 1.5. Let $P$ and $\bar{P}$ be projective structures over $M$. We say that $P$ and $\bar{P}$ are equivalent to each other if there exists a fibre-preserving diffeomorphism $J$ : $P \rightarrow \bar{P}$ such that

(i) $\left.J^{*} \Theta\right|_{\bar{p}}=\left.\Theta\right|_{p}$, and

(ii) $J$ induces identity on $M$.

The above definition can be slightly generalized. Suppose we have projective structures $P$ over $M$ and $\bar{P}$ over $\bar{M}$, another manifold of dimension $n$. Then we say that $P$ and $\bar{P}$ are equivalent to each other if there exists a fibre-preserving diffeomorphism $J: P \rightarrow \bar{P}$ such that $\left.J^{*} \Theta\right|_{\bar{p}}=\left.\Theta\right|_{p}$. Let $j$ be the diffeomorphism $M \rightarrow \bar{M}$ induced by $J$. Then replacing $M$ by $j(M)$ we obtain Definition 1.5.

2. Moving frames on submanifolds of $\mathbf{R} P^{n}$. In this section we construct (higher order) moving frames on submanifolds of $\mathbf{R} P^{n}$ following Cartan (see [1 and 2]). For a description of the general theory dealing with the problems of higher order contacts and frames of submanifolds of homogeneous spaces, we refer to [8].

Let $f: S^{p} \rightarrow \mathbf{R} P^{n}$ be a $p$-dimensional submanifold given locally as a graph $\left(x^{i}\right) \mapsto\left(x^{i}, f^{a}(x)\right)$, where $x=\left(x^{i}\right), 1 \leqslant i \leqslant p$, and $p+1 \leqslant a \leqslant n$. Let $h=\pi \circ I$ : $\operatorname{PGL}(n+1 ; \mathbf{R}) \rightarrow Q\left(\mathbf{R} P^{n}\right) \rightarrow L\left(\mathbf{R} P^{n}\right)$, i.e., $h\left(a^{\alpha}, a_{\beta}^{\alpha}, a_{\beta}\right)=\left(a^{\alpha}, a_{\beta}^{\alpha}\right) \equiv\left(a^{\alpha}, e_{\beta}\right)$.

Let $L_{0}=f^{-1} \operatorname{PGL}(n+1 ; \mathbf{R})$ be the pull-back bundle over $S$ :

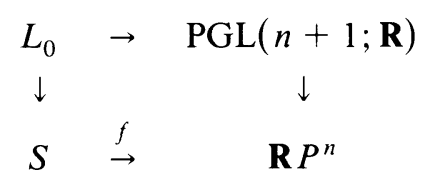

$L_{0}$ is a $G_{0}$-principal bundle, and a section a called a zeroth order moving frame field along $f$. Suppose we have a zeroth order moving frame field $u: S \rightarrow L_{0}$. This gives a local representation of the derivative map

$$
d f=\phi^{\alpha} \otimes e_{\alpha}=u^{*} \Omega^{\alpha} \otimes e_{\alpha} \in \Gamma\left(T^{*}(s) \otimes f^{-1} T\left(\mathbf{R} P^{n}\right)\right) .
$$

Consider $u_{0}: S \rightarrow L_{0}$ given by

$$
\left(x^{i}\right) \mapsto\left[\begin{array}{ccc}
1 & 0 & 0 \\
x^{1} & & \\
\vdots & I_{p} & \\
x^{p} & & \\
f^{p+1}(x) & & \\
\vdots & \left(\frac{\partial f^{a}}{\partial x^{i}}(x)\right) & I_{n-p} \\
f^{n}(x) & &
\end{array}\right]
$$


Then

$$
u_{0}^{*} \Omega^{a} \equiv\left(u_{0}^{-1} d u_{0}\right)_{0}^{a}=-\frac{\partial f^{a}}{\partial x^{k}} d x^{k}+d f^{a}=0 .
$$

Hence $u_{0}$ normalizes $d f$ in the sense that

$$
d f=\phi^{1} \otimes e_{1} \oplus \cdots \oplus \phi^{p} \otimes e_{p},
$$

where $\left(\phi^{i}=u_{0}^{*} \Omega^{i}\right)$ is a coframe field on $S$. (We obtained so-called "first order normal form".)

Suppose we have another zeroth order moving frame field $u: S \rightarrow L_{0}$. Then $u=u_{0} \cdot g$, where $g: S \rightarrow G_{0}$ is some smooth map. Consider the group action of $G_{0}$ on $\mathfrak{M}_{0}=\mathbf{R}^{n}$ defined by the equation $u^{*}\left(\Omega^{\alpha}\right)=\left(u_{0} \cdot g\right)^{*}\left(\Omega^{\alpha}\right)$. Using the fact that

$$
\left(u_{0} \cdot g\right)^{*}\left(\Omega^{\alpha}\right)=\Re_{0} \text {-component of } \operatorname{Ad}\left(g^{-1}\right) u_{0}^{*} \Omega,
$$

we compute: if $g=\left(a_{\beta}^{\alpha}, a_{\beta}\right)=\left(A, a_{\beta}\right) \in G_{0}$, and $v \in \mathbf{R}^{n}$, then the action is given by

$$
v \mapsto A v .
$$

The isotropy group for this action is

$$
G_{1}=\left\{\left[\begin{array}{ccc}
1 & \left(a_{j}\right) & \left(\eta_{b}\right) \\
0 & \left(a_{j}^{i}\right) & \left(\eta_{b}^{i}\right) \\
0 & 0 & \left(b_{b}^{a}\right)
\end{array}\right]: \begin{array}{l}
\left(a_{j}^{i}\right) \in \mathrm{GL}(p ; \mathbf{R}), \\
{ }^{\mathrm{t}}\left(a_{j}\right) \in \mathbf{R}^{p},{ }^{\mathrm{t}}\left(\eta_{b}\right) \in \mathbf{R}^{n-p}, . \\
\left(\eta_{b}^{i}\right) \in \mathbf{R}^{p x(n-p)},\left(b_{b}^{a}\right) \in \mathrm{GL}(n-p ; \mathbf{R})
\end{array}\right\} .
$$

DEFINITION 2.6. The bundle of first order moving frames of $f$ is $L_{1}=\left\{u_{0} \cdot G_{1}\right\}$, where $u_{0}$ is given by (2.2). Any section $u: S \rightarrow L_{1}$ is called a first order moving frame field along $f$. We emphasize that any first order moving frame field normalizes $d f$ in the sense defined by (2.3).

On $L_{1}, \Omega^{a}=0$ for $p+1 \leqslant a \leqslant n$. Differentiating both sides of the equation and using the structure equation, we obtain

$$
\Omega_{i}^{a} \wedge \Omega^{i}=0
$$

By Cartan's lemma it follows that

$$
\Omega_{j}^{a}=A_{j k}^{a} \Omega^{k},
$$

where $A_{j k}^{a}=A_{k j}^{a}$ are functions on $L_{1}$.

Though the technique in what follows is quite general, we restrict ourselves to the case of surfaces in $\mathbf{R} P^{3}$ from now on to ease computational difficulties and to simplify exposition. Then (2.7) becomes

$$
\Omega_{1}^{3}=A_{11} \Omega^{1}+A_{12} \Omega^{2} \text { and } \Omega_{2}^{3}=A_{12} \Omega^{1}+A_{22} \Omega^{2} .
$$

Let

$$
E_{4}=\left|\begin{array}{llll}
0 & 0 & 0 & 0 \\
0 & 0 & 0 & 0 \\
0 & 0 & 0 & 0 \\
0 & 1 & 0 & 0
\end{array}\right| \text { and } E_{5}=\left|\begin{array}{cccc}
0 & 0 & 0 & 0 \\
0 & 0 & 0 & 0 \\
0 & 0 & 0 & 0 \\
0 & 0 & 1 & 0
\end{array}\right|
$$


Then we have a vector space direct sum decomposition $\mathfrak{g}_{0}=\mathfrak{g}_{1} \oplus \mathfrak{⿰} \mathfrak{K}_{1}$, where $\mathfrak{g}_{0}$ and $\Omega_{1}$ are Lie algebras of $G_{0}$ and $G_{1}$, respectively, and $\Omega_{1}=\operatorname{span}\left\{E_{4}, E_{5}\right\}$. We write $\left(\Omega_{j}^{3}\right)=\Omega_{1}^{3} \otimes E_{4} \oplus \Omega_{2}^{3} \otimes E_{5}=\Re_{1}$-component of $\Omega$.

Fix a first order moving frame field $\hat{u}: S \rightarrow L_{1}$. Write $\hat{u}^{*} \Omega_{j}^{3}=\hat{x}_{j k} \hat{u}^{*} \Omega^{h}$ for some functions $\hat{x}_{j k}$ on $S$. Suppose $u: S \rightarrow L_{1}$ is given by $u=\hat{u} \cdot g$ for a smooth map $g$ : $S \rightarrow G_{1}$. Let $x_{j k}$ be functions so that $u^{*} \Omega_{j}^{3}=x_{j k} u^{*} \Omega^{k}$. We will compute the action of $G_{1}$ on $\left(\hat{x}_{j k}\right)$. Observe that

$$
\begin{aligned}
u^{*}\left(\left(\Omega^{\alpha}\right) \oplus\left(\Omega_{j}^{3}\right)\right) & =(\hat{u} \cdot g) *\left(\left(\Omega^{\alpha}\right) \oplus\left(\Omega_{j}^{3}\right)\right) \\
& =\mathfrak{M}_{0} \oplus \boldsymbol{M}_{1} \text {-component of } \operatorname{Ad}\left(g^{-1}\right) \hat{u}^{*} \Omega .
\end{aligned}
$$

Some matrix multiplications show that

$$
X=g(\hat{X})=b^{-1} \mathrm{t} A \hat{X} A,
$$

where

$$
g=\left[\begin{array}{ccc}
1 & \left(a_{j}\right) & \eta^{0} \\
0 & A & \left(\eta^{i}\right) \\
0 & 0 & b
\end{array}\right] \in G_{1}, \quad X=\left(x_{j k}\right) \text { and } \hat{X}=\left(\hat{x}_{j k}\right)
$$

There are four orbits of this action represented by

$$
\left|\begin{array}{ll}
0 & 0 \\
0 & 0
\end{array}\right|,\left|\begin{array}{ll}
1 & 0 \\
0 & 0
\end{array}\right|, \quad\left|\begin{array}{ll}
1 & 0 \\
0 & 1
\end{array}\right| \text { and }\left|\begin{array}{ll}
0 & 1 \\
1 & 0
\end{array}\right| .
$$

Assuming $f$ is of constant orbit type, we call the first orbit type degenerate type, and the rest are called parabolic type, elliptic type and hyperbolic type, respectively.

Suppose $f$ is of degenerate type. Then the isotropy subgroup $G_{2}$ of the action (2.9) is $G_{1}$ itself, and $L_{1}$ is the bundle of Frenet frames. On $L_{1}, \Omega^{3}=\Omega_{1}^{3}=\Omega_{2}^{3}=0$. Consider the involutive left-invariant distribution on $\operatorname{PGL}(4 ; \mathbf{R})$ defined by the exterior differential system $\left\{\Omega^{3}=\Omega_{1}^{3}=\Omega_{2}^{3}=0\right\}$. The analytic subgroup of PGL $(4 ; \mathbf{R})$ corresponding to this distribution is

$$
H=\left\{\left[\begin{array}{ccc}
1 & \left(a_{j}\right) & \eta^{0} \\
\left(a^{i}\right) & A & \left(\eta^{i}\right) \\
0 & 0 & b
\end{array}\right] \in \operatorname{PGL}(4 ; \mathbf{R})\right\}
$$

Hence $f(s) \subseteq H / G_{0} \cap H \simeq \mathbf{R} P^{2}$

We characterize the remaining (constant) type surfaces by the following theorem.

THeOREM 2.11. Let $\left(x^{1}, x^{2}, f(x)\right)$ be a surface in $\mathbf{R} P^{3}$, and let

$$
f_{i j}(x)=\partial^{2} f(x) / \partial x^{i} \partial x^{i}
$$

Then:

(i) $f \in$ parabolic type if and only if $\operatorname{rank}\left(f_{i j}\right) \equiv 1$;

(ii) $f \in$ elliptic type if and only if $\operatorname{rank}\left(f_{i j}\right) \equiv 2$ and $\operatorname{det}\left(f_{i j}\right)>0$ : and

(iii) $f \in$ hyperbolic type if and only if $\operatorname{rank}\left(f_{i j}\right) \equiv 2$ and $\operatorname{det}\left(f_{i j}\right)<0$. 
Proof. Let

$$
u_{0}=\left[\begin{array}{cccc}
1 & 0 & 0 & 0 \\
x^{1} & & & 0 \\
x^{2} & I_{2} & & 0 \\
f(x) & f_{1}(x) & f_{2}(x) & 1
\end{array}\right]: S \rightarrow L_{1},
$$

where $f_{i}(x)=\partial f(x) / \partial x^{i}$. Then

$$
u_{0}^{-1}=\left[\begin{array}{cccc}
1 & 0 & 0 & 0 \\
-x^{1} & & & 0 \\
-x^{2} & I_{2} & & 0 \\
\Delta_{f}(x) & -f_{1}(x) & -f_{2}(x) & 1
\end{array}\right],
$$

where $\Delta_{f}(x)=f_{k}(x) x^{k}-f(x)$. We compute the following:

$$
\begin{aligned}
& u_{0}^{*} \Omega^{i}=\left(u_{0}^{-1} d u_{0}\right)_{0}^{i}=d x^{i}, \\
& u_{0}^{*} \Omega_{j}^{i}=\left(u_{0}^{-1} d u_{0}\right)_{j}^{i}=0, \\
& u_{0}^{*} \Omega_{j}^{3}=\left(u_{0}^{-1} d u_{0}\right)_{j}^{3}=d f_{j}=f_{j k} d x^{k},
\end{aligned}
$$

so $u_{0}^{*} \Omega_{j}^{3}=f_{j k} u_{0}^{*} \Omega^{k}$. Comparing this expression with that immediately preceding (2.8), the rest follows.

Some obvious examples of parabolic, elliptic and hyperbolic type surfaces are given by $f\left(x^{1}, x^{2}\right)=\left(x^{1}\right)^{2}, f\left(x^{1}, x^{2}\right)=\left(x^{1}\right)^{2}+\left(x^{2}\right)^{2}$, and $f\left(x^{1}, x^{2}\right)=\left(x^{1}\right)^{2}-$ $\left(x^{2}\right)^{2}$, respectively. To obtain Frenet frames we need to construct fourth order moving frames in general. However, for the rest of this paper what we have so far is sufficient.

3. Induced projective structures. In contrast to Riemannian geometry, given a submanifold in $\mathbf{R} \boldsymbol{P}^{n}$ there are, in general, not one but many distinct induced projective structures on it. Roughly speaking, a choice of normal framing determines an induced structure. In this section we describe the totality of such structures.

Let $f: S^{p} \rightarrow \mathbf{R} P^{n}$ be a $p$-dimensional submanifold given locally as a graph as in $\S 2$. Recall that the bundle of first order moving frames of $f$ is $L_{1}=\left\{u_{0} \cdot G_{1}\right\}$ (see Definition 2.6):

Let

$$
H=\left\{\left[\begin{array}{ccc}
1 & \left(a_{j}\right) & 0 \\
0 & \left(a_{j}^{i}\right) & 0 \\
0 & 0 & I_{n-p}
\end{array}\right]: \begin{array}{l}
\left(a_{j}^{i}\right) \in \mathrm{GL}(p ; \mathbf{R}), \\
{ }^{\mathrm{t}}\left(a_{j}\right) \in \mathbf{R}^{p}
\end{array}\right\} .
$$

be a subgroup of $G_{1}$ and

$$
N=\left\{\left[\begin{array}{cc} 
& \left(\eta_{b}\right) \\
I_{p+1} & \left(\eta_{b}^{i}\right) \\
0 & \left(b_{b}^{a}\right)
\end{array}\right]: \begin{array}{l}
\mathrm{t}\left(\eta_{b}\right) \in \mathbf{R}^{n-p}\left(b_{b}^{a}\right) \in \mathrm{GL}(n-p ; \mathbf{R}), \\
\left(\eta_{b}^{i}\right) \in \mathbf{R}^{p \times(n-p)}
\end{array}\right\}
$$


be a normal subgroup of $G_{1}$. Then $G_{1}=H \cdot N$, a semidirect product, and we identify $G_{1} / N$ with $H$. Observe that $H$ is isomorphic to the projective isotropy group.

Consider the quotient space $L_{1} / N$. Because $N$ is a normal subgroup of $G_{1}$, it follows from the general theory of principal bundles that $L_{1} / N \rightarrow S$ is a principal $G_{1} / N=H$ bundle. Furthermore, since $H$ is isomorphic to the projective isotropy group of $\mathbf{R} P^{p}$, this bundle $L_{1} / N \rightarrow S$ is a candidate for defining a projective structure on $S$, provided there is some natural way to imbed the bundle $L_{1} / N \rightarrow S$ into $Q(S) \rightarrow S$. As a first step in this process we construct a natural imbedding of $L_{1} / N \rightarrow S$ into $L_{1} \rightarrow S$ such that the map $L_{1} / N \rightarrow L_{1}$ is a section of the principal $N$-bundle $L_{1} \rightarrow L_{1} / N$. Each such imbedding will be constructed from a "normal frame field".

As a local coordinate system in $L_{1} / N$ we use the restriction of the coordinates $\left(a^{\alpha}, a_{\beta}^{\alpha}, a_{\beta}\right)$ on $\operatorname{PGL}(n+1 ; \mathbf{R})$ and we call them $\left(b^{i}, b_{j}^{i}, b_{j}\right)$. Then the right action of $H$ is given locally by

$$
\left(b^{i}, b_{j}^{i}, b_{j}\right)\left(h_{j}^{i}, h_{j}\right)=\left(b^{i}, b_{k}^{i} h_{j}^{i}, h_{j}+b_{k} h_{j}^{k}\right),
$$

where $\left(h_{j}^{i}, h_{j}\right) \in H$.

We fix the following sections:

$$
\begin{gathered}
q_{0}: S \rightarrow L_{1} / N, \quad \text { where } q_{0}(x)=\left(x^{i}, \delta_{j}^{i}, 0\right) \\
\hat{i}: L_{1} / N \rightarrow L_{1}, \quad \text { where } \hat{i}\left(x^{i}, b_{j}^{i}, b_{j}\right)=u_{0}(x) \cdot\left[\begin{array}{ccc}
1 & \left(b_{j}\right) & 0 \\
0 & \left(b_{j}^{i}\right) & 0 \\
0 & 0 & I_{n-p}
\end{array}\right]
\end{gathered}
$$

i.e.,

$$
(\hat{i})_{b}^{A}=\left(\begin{array}{c}
0 \\
I_{n-p}
\end{array}\right), \quad 0 \leqslant A \leqslant n .
$$

Observe that $\hat{i} \circ q_{0}=u_{0}$.

The following two lemmas are straightforward.

LEMMA 3.4. Any section $i: L_{1} / N \rightarrow L_{1}$ is given by $i=\hat{i} \cdot \tilde{\eta}$ for some map $\tilde{\eta}$ : $L_{1} / N \rightarrow N$.

Proof. The structure group of $L_{1} \rightarrow L_{1} / N$ is $N$.

LEMmA 3.5. Any map $\eta: S \rightarrow N$ determines a map $\tilde{\eta}: L_{1} / N \rightarrow N$ such that $\tilde{\eta} \circ q_{0}=\eta$.

Proof. Put $\tilde{\eta} \circ q_{0}(x)=\eta(x)$ for every $x \in S$. This defines $\tilde{\eta}$ at one point of each fibre of $L_{1} / N \rightarrow S$. To extend $\tilde{\eta}$ along the fibre through $q_{0}(x)$, put $\tilde{\eta}\left(q_{0}(x) \cdot h\right)=$ $h^{-1} \cdot \eta(x) \cdot h$ for $h \in H$.

We now have the following proposition.

Proposition 3.6. Any map $\eta: S \rightarrow N$ determines a bundle map section $i_{\eta}: L_{1} / N \rightarrow$ $L_{1}$ by $i_{\eta}=\hat{i} \cdot \hat{\eta} \cdot \tilde{\eta}$, where $\tilde{\eta}$ is given by Lemma 3.5 . 
Proof. $i_{\eta}(q)=\hat{i}(q) \cdot \tilde{\eta}(q)$, for $q \in L_{1} / N$, defines a map $L_{1} / N \rightarrow L_{1}$. Clearly this is a section. For $q \in L_{1} / N$ choose $x \in S$ and $h_{0} \in H$ so that $q=q_{0}(x) \cdot h_{0}$. Then

$$
\begin{aligned}
i_{\eta}(q \cdot h) & =\hat{i}(q \cdot h) \cdot \tilde{\eta}\left(q_{0}(x) \cdot h_{0} \cdot h\right) \\
& =\hat{i}(q) \cdot h \cdot\left(h_{0} \cdot h\right)^{-1} \cdot \eta(x) \cdot\left(h_{0} \cdot h\right) \\
& =\hat{i}(q) \cdot h_{0}^{-1} \cdot \eta(x) \cdot h_{0} \cdot h=\hat{i}(q) \cdot \tilde{\eta} \cdot\left(q_{0}(x) \cdot h_{0}\right) \cdot h=i_{\eta}(q) \cdot h .
\end{aligned}
$$

Thus $i_{\eta}$ is a bundle map, where the group map is given by the inclusion.

The following lemma is technically important.

LEMMA 3.7. Any first order moving frame field $u: S \rightarrow L_{1}$ can be written as $u=i_{\eta} \circ q$ for some $\eta: S \rightarrow N$ and $q: S \rightarrow L_{1} / N$.

Proof. We can write $u=u_{0} \cdot g$, where $g: S \rightarrow G_{1}$ a smooth map. Now, $G_{1}=$ $H \cdot N$, and $N$ is normal in $G_{1}$. Thus there exist smooth maps $h: S \rightarrow H$ and $\eta: S \rightarrow N$ so that $\eta \cdot h=g$. Let $q=q_{0} \cdot h$. Then

$$
i_{\eta} \circ q=\left(\hat{i} \circ q_{0}\right) \cdot \eta \cdot h=\left(\hat{i} \circ q_{0}\right) \cdot g=u_{0} \cdot g \text {. }
$$

We now write some explicit local expressions for the case $p=2$ and $n=3$, i.e., surfaces in $\mathbf{R} P^{3}$. Let $\eta: S \rightarrow N$ be given by

$$
\eta(x)=\left[\begin{array}{cccc} 
& & & \eta^{0}(x) \\
I_{3} & & & \eta^{i}(x) \\
0 & 0 & 0 & b(x)
\end{array}\right]
$$

Then $i_{\eta}: L_{1} / N \rightarrow L_{1}$ is given by

$$
\left(x^{i}, b_{j}^{i}, b_{j}\right) \mapsto\left(a^{\alpha} \circ i_{\eta}, a_{\beta}^{\alpha} \circ i_{\eta}, a_{\beta} \circ i_{\eta}\right),
$$

where

$$
\begin{aligned}
& \left(a^{\alpha} \circ i_{\eta}\right)=\left(\begin{array}{c}
x^{i} \\
f(x)
\end{array}\right), \quad a_{j}^{i} \circ i_{\eta}=b_{j}^{i}, \quad a_{3}^{i} \circ i_{\eta}=\eta^{i}, \quad a_{j}^{3} \circ i_{\eta}=f_{k} b_{j}^{k}, \\
& a_{3}^{3} \circ i_{\eta}=f_{k} \eta^{k}+b, \quad a_{j} \circ i_{\eta}=b_{j} \text { and } a_{3} \circ i_{\eta}=\eta^{0} \text {. }
\end{aligned}
$$

In terms of matrices,

$$
i_{\eta}\left(x^{i}, b_{j}^{i}, b_{j}\right)=\left[\begin{array}{ccc}
1 & \left(b_{j}\right) & \eta^{0}(x) \\
\left(x^{i}\right) & x^{i} b_{j}+b_{j}^{i} & x^{i} \eta^{0}(x)+\eta^{i}(x) \\
f(x) & f(x) b_{j}+f_{k}(x) \cdot b_{j}^{k} & b+f_{k}(x) \eta^{k}(x)+f(x) \eta^{0}(x)
\end{array}\right]
$$

Observe that $i_{\eta}\left(L_{1} / N\right) \subseteq L_{1}$. We also have the following computations:

$$
\begin{aligned}
& i_{\eta}^{*} \Omega^{i}=\left(b^{-1}\right)_{k}^{i} d x^{k}, \\
& i_{\eta}^{*} \Omega_{j}^{i}=\left(b^{-1}\right)_{k}^{i} b_{j} d x^{k}+\left(b^{-1}\right)_{k}^{i} d b_{j}^{k}-\left(\eta^{i} / b\right)\left(b^{-1}\right)_{l}^{i} b_{j}^{k} d f_{k}+\delta_{j}^{i} b_{l}\left(b^{-1}\right)_{k}^{l} d x^{k}, \\
& i_{\eta}^{*} \Omega_{j}^{3}=b^{-1} b_{j}^{l} d f_{l} .
\end{aligned}
$$

Observe that in the above expressions $\eta^{0}$ does not appear. 
Going back to the general case, we let $f^{-1} Q\left(\mathbf{R} P^{n}\right)$ be the pull-back bundle. Then there is a natural imbedding $L_{1} \rightarrow f^{-1} Q\left(\mathbf{R} P^{n}\right)$ :

$$
\begin{array}{ccc}
\operatorname{PGL}(n+1 ; \mathbf{R}) & \stackrel{I^{\bullet}}{\rightarrow} & Q\left(\mathbf{R} P^{n}\right) \\
\uparrow & & \uparrow \\
f^{-1} \operatorname{PGL}(n+1 ; \mathbf{R})=L_{0} \supset L_{1} & \rightarrow & f^{-1} Q\left(\mathbf{R} P^{n}\right)
\end{array}
$$

Given $i_{\eta}: L_{1} / N \rightarrow L_{1}$, define $I_{\eta}: L_{1} / N \rightarrow f^{-1} Q\left(\mathbf{R} P^{n}\right)$ by the following commutative diagram:

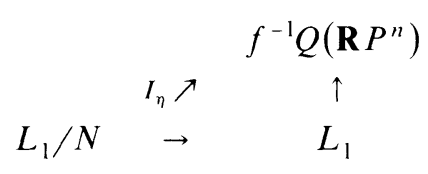

We then have the following propositions.

Proposition 3.10. (i)

$$
\left.I_{\eta}^{*} \Theta^{\prime}\right|_{f^{-1} Q\left(\mathbf{R} P^{n}\right)}=\left.i_{\eta}^{*}\left(\Omega^{i}, \Omega_{j}^{i}\right)\right|_{L_{1}},
$$

where $\Theta^{\prime}=\left(\theta^{i}, \theta_{j}^{i}\right)=\mathbf{R}^{p} \oplus \mathfrak{g l}(p ; \mathbf{R})$-component of the canonical form $\Theta$ of $Q\left(\mathbf{R} P^{n}\right)$, and

(ii)

$$
\left.I_{\eta}^{*} \Theta^{a}\right|_{f^{-1} Q\left(\mathbf{R} P^{n}\right)}=0 \text { for } p+1 \leqslant a \leqslant n .
$$

Proposition 3.11. There is a naturally defined bundle imbedding $\Phi_{\eta}: L_{1} / N \rightarrow Q(S)$ with the group map $H \rightarrow G^{2}(p)$ given by $\left(h_{j}^{i}, h_{j}\right) \mapsto\left(h_{j}^{i},-\left(h_{j}^{i} h_{k}+h_{k}^{i} h_{j}\right)\right)$ so that

$$
\Phi_{\eta}^{*} \Theta_{Q(S)}=\left.I_{\eta}^{*} \Theta^{\prime}\right|_{f^{-1} Q\left(\mathbf{R} P^{n}\right)},
$$

where $\Theta_{Q(S)}$ is the canonical form of $Q(S)$. If $p=2$ and $n=3, \Phi_{\eta}$ is given explicitly by

$$
\Phi_{\eta}\left(x^{i}, b_{j}^{i}, b_{j}\right)=\left(x^{i}, b_{j}^{i},-\left(b_{j}^{i} b_{k}+b_{k}^{i} b_{j}\right)+\left(\eta^{i} / b\right) f_{n m} b_{j}^{n} b_{k}^{m}\right) .
$$

We end this section with the definition.

DeFINITION 3.12. An induced projective structure on $f$ is a pair $\left(L_{1} / N, I_{\eta}\right)$.

4. Equivalence problems for submanifolds in $\mathbf{R} P^{n}$. In this section we derive exterior differential systems for extrinsic equivalences. We give the result for some special cases; the detailed computations dealing with general cases will appear in a forthcoming paper.

Let $f, \bar{f}: S^{p} \rightarrow \mathbf{R} P^{n}$ be two submanifolds and $\eta, \bar{\eta}: S \rightarrow N$ maps as before.

DEFINITION 4.1. $f$ and $\bar{f}$ are (projectively) equivalent to each other with respect to the pair $(\eta, \bar{\eta})$ if the projective structures $\left(L_{1} / N, I_{\eta}\right)$ and $\left(\bar{L}_{1} / N, \bar{I}_{\bar{\eta}}\right)$ are equivalent as in Definition 1.5.

Suppose $f$ ard $\bar{f}$ are projectively equivalent w.r.t. $(\eta, \bar{\eta})$. Then given any section $q$ : $S \rightarrow L_{1} / N$, there exists a corresponding section $\bar{q}: S \rightarrow \bar{L}_{1} / N$ defined by $\bar{q}: J \circ q$, and we have, by Proposition 3.10 and the fact that $J$ gives a bundle equivalence,

$$
\left.q^{*} \circ i_{\eta}^{*}\left(\Omega^{i}, \Omega_{j}^{i}\right)\right|_{L_{1}}=\left.\bar{q}^{*} \circ i_{\bar{\eta}}^{*}\left(\Omega^{i}, \Omega_{j}^{i}\right)\right|_{L_{1}},
$$


where $i_{\eta}$ is given by Proposition 3.6:

$$
\begin{array}{ccc}
L_{1} / N & \stackrel{J}{\rightarrow} & \bar{L}_{1} / N \\
q \nwarrow & & \nearrow \bar{q} \\
& S &
\end{array}
$$

The following lemma is technically important.

LEMMA 4.3. $f$ and $\bar{f}$ are equivalent to each other w.r.t. $(\eta, \bar{\eta})$ if and only if there exists a smooth map $h: S \rightarrow H$ such that

$$
\left.q_{0}^{*} \circ i_{\eta}^{*}\left(\Omega^{i}, \Omega_{j}^{i}\right)\right|_{L_{1}}=\left.\left(\bar{q}_{0} \cdot h\right) * \circ i_{\bar{\eta}}^{*}\left(\Omega^{i}, \Omega_{j}^{i}\right)\right|_{L_{1}},
$$

where $q_{0}$ is the zero section so that $u_{0}=\hat{i} \circ q_{0}$ (see (3.3)).

Proof. Necessity follows from (4.2). To prove the converse, let $\omega^{i}=\left.i_{\eta}^{*} \Omega^{i}\right|_{L}$, $\omega_{j}^{i}=\left.i_{\eta}^{*} \Omega_{j}^{i}\right|_{L_{1}}$ and similarly for barred quantities. We then assume there is a map $h$ : $S \rightarrow H$ such that

$$
q_{0}^{*}\left(\omega^{i}, \omega_{j}^{i}\right)=\left(\bar{q}_{0} \cdot h\right) *\left(\bar{\omega}^{i}, \bar{\omega}_{j}^{i}\right) .
$$

Define a fibre-preserving diffeomorphism $J: L_{1} / N \rightarrow \bar{L}_{1} / N$ so that $J \circ q_{0}=\bar{q}_{0} \cdot h$. (This can always be done locally since $L_{1} / N$ and $\bar{L}_{1} / N$ are isomorphic bundles.) Since $\left(\omega^{i}\right)$ and $\left(\bar{\omega}^{i}\right)$ vanish on vertical vectors and $J$ is fibre-preserving, it follows that $J^{*}\left(\bar{\omega}^{i}\right)-\left(\omega^{i}\right)$ also vanish on vertical vectors. That is, $J^{*}\left(\bar{\omega}^{i}\right)-\left(\omega^{i}\right)$ are linear combinations of $\left(\omega^{i}\right)$. Then by (4.5) they must in fact be 0 since $q_{0}^{*}\left(\omega^{i}\right)$ form a coframe field on $S$. Differentiate both sides of the equations $J^{*}\left(\bar{\omega}^{i}\right)-\left(\omega^{i}\right)=0$ and obtain

$$
J^{*}\left(-\bar{\omega}_{k}^{i} \wedge \bar{\omega}^{k}\right)=\left(-\omega_{k}^{i} \wedge \omega^{k}\right) .
$$

By Cartan's lemma $\left(\omega_{j}^{i}-J^{*} \bar{\omega}_{j}^{i}\right)$ must be linear combinations of $\left(\omega^{i}\right)$. But

$$
q_{0}^{*}\left(\omega_{j}^{i}-J^{*} \bar{\omega}_{j}^{i}\right)=0
$$

and observing once again that $q_{0}^{*}\left(\omega^{i}\right)$ form a coframe field on $S$, the proof is complete.

We now characterize the so-called normal projective connection as a preserved quantity under equivalence. We do this for the case where $p=2$ and $n=3$, the general case being similar. Let $f, \bar{f}: S \rightarrow \mathbf{R} P^{3}$ be surfaces. We then have

LemMa 4.7. Let $i_{\eta}: L_{1} / N \rightarrow L_{1}$ be any bundle map section defined by Proposition 3.6. Then

$$
\left.i_{\eta}^{*} \Omega_{3}^{A}\right|_{L_{1}} \in \operatorname{span}\left\{\left.i_{\eta}^{*} \Omega^{1}\right|_{L_{1}},\left.i_{\eta}^{*} \Omega^{2}\right|_{L_{1}}\right\}
$$

where $0 \leqslant A \leqslant 3$.

Proof. The structure groups for $L_{1} / N$ and $L_{1}$ are $H$ and $G=H \cdot N$, respectively. We observe that the map $i_{\eta}$ restricted to the group $H$ is the identity, and $i_{\eta}(q \cdot h)=$ $i_{\eta}(q) \cdot h$ for every $q \in L_{1} / N$ and $h \in H$. The rest is easy. 
TheOREM 4.8 (NoRMAl PROJECTIVE CONNECTION). Let $\left(L_{1} / N, I_{\eta}\right)$ be an induced projective structure on $f$. Also let $\left(\bar{L}_{1} / N, \bar{I}_{\bar{\eta}}\right)$ be any induced projective structure on $\bar{f}$ equivalent to $\left(L_{1} / N, I_{\eta}\right)$. Then $\left(\omega^{i}, \omega_{j}^{i}\right)$ and $\left(\bar{\omega}^{i}, \bar{\omega}_{j}^{i}\right)$ (notation from (4.5)) can be uniquely enlarged to Cartan connections $\left(\omega^{i}, \omega_{j}^{i}, \hat{\omega}_{j}\right)$ and $\left(\bar{\omega}^{i}, \bar{\omega}_{j}^{i}, \hat{\bar{\omega}}_{j}\right)$ such that $\bar{q}^{*}\left(\bar{\omega}^{i}, \bar{\omega}_{j}^{i}, \hat{\bar{\omega}}_{j}\right)=q^{*}\left(\omega^{i}, \omega_{j}^{i}, \hat{\omega}_{j}\right)$ for every $(q, \bar{q})$ as in $(4.2)$.

Proof. From (4.2) it follows that the exterior differential system $\left\{\omega^{i}=\bar{\omega}^{i}\right.$, $\left.\omega_{j}^{i}=\bar{\omega}_{j}^{i}, i, j=1,2\right\}$ on $L_{1} / N \times \bar{L}_{1} / N$ has solutions of the form $(q, \bar{q}): S \rightarrow L_{1} / N$ $\times \bar{L}_{1} / N$. Using the previous lemma, we let $\left.i_{\eta}^{*}\left(\Omega_{3}^{i} \wedge \Omega_{j}^{3}\right)\right|_{L_{1}}=-K_{j}^{i} \omega^{1} \wedge \omega^{2}$ and similarly for barred quantities, where $K_{j}^{i}$ and $\bar{K}_{j}^{i}$ are functions on $L_{1} / N$ and $\bar{L}_{1} / N$, respectively. We close the system and write the quadratic equations modulo the system.

$$
\begin{aligned}
& \omega^{1} \wedge\left(\omega_{2}-\bar{\omega}_{2}\right)-\left(K_{2}^{1}-\bar{K}_{2}^{1}\right) \omega^{1} \wedge \omega^{2} \equiv 0, \\
& \omega^{2} \wedge\left(\omega_{1}-\bar{\omega}_{1}\right)-\left(K_{1}^{2}-\bar{K}_{1}^{2}\right) \omega^{1} \wedge \omega^{2} \equiv 0, \\
& 2 \omega^{1} \wedge\left(\omega_{1}-\bar{\omega}_{1}\right)+\omega^{2} \wedge\left(\omega_{2}-\bar{\omega}_{2}\right)-\left(K_{1}^{1}-\bar{K}_{1}^{1}\right) \omega^{1} \wedge \omega^{2} \equiv 0, \\
& \omega^{1} \wedge\left(\omega_{1}-\bar{\omega}_{1}\right)+2 \omega^{2} \wedge\left(\omega_{2}-\bar{\omega}_{2}\right)-\left(K_{2}^{2}-\bar{K}_{2}^{2}\right) \omega^{1} \wedge \omega^{2} \equiv 0,
\end{aligned}
$$

where $\omega_{j}=\left.i_{\eta}^{*} \Omega_{j}\right|_{L_{1}}$, etc.

We have two unknowns, $\omega_{1}-\bar{\omega}_{1}$ and $\omega_{2}-\bar{\omega}_{2}$, and four equations. Hence the system is not in involution. We let

$$
\begin{aligned}
& \hat{\omega}_{1}=\omega_{1}+\left(K_{1}^{2} \omega^{1}+\frac{1}{3}\left(K_{2}^{2}-2 K_{1}^{1}\right) \omega^{2}\right), \\
& \hat{\omega}_{2}=\omega_{2}-\left(\frac{1}{3}\left(K_{1}^{1}-2 K_{2}^{2}\right) \omega^{1}+K_{2}^{1} \omega^{2}\right),
\end{aligned}
$$

and similarly for barred quantities.

Then (4.9) becomes

$$
\omega^{i} \wedge\left(\hat{\omega}_{j}-\hat{\bar{\omega}}_{j}\right) \equiv 0
$$

By Cartan's lemma it follows that we must prolong the system by adding equations $\hat{\omega}_{j}-\hat{\bar{\omega}}_{j}=0, j=1,2$. That is to say, $q^{*}\left(\hat{\boldsymbol{\omega}}_{j}\right)=\bar{q}^{*}\left(\hat{\bar{\omega}}_{j}\right)$ for every solution $(q, \bar{q})$ : $S \rightarrow L_{1} / N \times \bar{L}_{1} / N$ of the system.

Observe that (4.10) gives the explicit expression for the normal projective connection induced by $\eta$. The following remark is crucial.

REMARK 4.12. The $\mathfrak{R}_{0}^{*}$-component of the normal projective connection associated to an induced projective structure does not, in general, arise as a restriction of the Maurer-Cartan form.

Recall that if $f: S \rightarrow \mathbf{R} P^{3}$ is of degenerate type (i.e., planar type), then $d f_{j} \equiv 0$. From (3.9) it follows that $\left.i_{\eta}^{*}\left(\Omega^{i}, \Omega_{j}^{i}\right)\right|_{L_{1}}$ are independent of the choices of normal vector fields. The following theorem is immediate.

THEOREM 4.13. If $f: S \rightarrow \mathbf{R} P^{3}$ is planar, then the only induced projective structure one obtains is the flat projective structure. Furthermore, the normal projective connection is given by $\left.\hat{i}^{*}\left(\Omega^{i}, \Omega_{j}^{i}, \Omega_{j}\right)\right|_{L_{1}}$.

We will now show that all nondegenerate surfaces are projectively equivalent to each other in the sense that, up to equivalence, the totality of induced structures does not depend on the particular imbedding of $S$ into $\mathbf{R} P^{3}$. 
THeOREM 4.14 (EXTRINSIC EQUivalenCE). Let $f, \bar{f}: S \rightarrow \mathbf{R} P^{3}$ be nonplanar real analytic surfaces and $\eta: S \rightarrow N$ a normal vector field on $f$. Then there always exists $\bar{\eta}(\eta): S \rightarrow N$, a normal vector field on $\bar{f}$ so that $f$ and $\bar{f}$ are projectively equivalent to each other w.r.t. $(\eta, \bar{\eta})$.

Proof. Let $u_{\eta}=i_{\eta} \circ q_{0}: S \rightarrow L_{1}$, and $\left.u_{\eta}^{*}\left(\Omega^{\alpha}, \Omega_{\beta}^{\alpha}, \Omega_{\beta}\right)\right|_{L_{1}}=\left(\phi^{\alpha}, \phi_{\beta}^{\alpha}, \phi_{\beta}\right)$. Then $\phi^{3}=$ 0 , and $\phi^{1}, \phi^{2}$ form a coframe field on $S$. We let $\left(a_{\beta k}^{\alpha}\right)$ and $\left(a_{\beta k}\right)$ be functions on $S$ such that $\phi_{\beta}^{\alpha}=a_{\beta k}^{\alpha} \phi^{k}$ and $\phi_{\beta}=a_{\beta k} \phi^{k}$. Let $\left.\left(\Omega^{\alpha}, \Omega_{\beta}^{\alpha}, \Omega_{\beta}\right)\right|_{L_{1}}=\left(\omega^{\alpha}, \omega_{\beta}^{\alpha}, \omega_{\beta}\right)$ and recall from (2.7) that $\omega_{1}^{3}=x \omega^{1}+y \omega^{2}$ and $\omega_{2}^{3}=y \omega^{1}+z \omega^{2}$, where $x, y, z$ are functions on $\overline{L_{1}}$.

Consider the exterior differential system on $S \times \bar{L}_{1}$,

$$
\Sigma:\left\{\phi^{i}=\omega^{i}, \phi_{j}^{i}=\omega_{j}^{i}, i, j=1,2\right\}
$$

with specified independent variables $\phi^{1}$ and $\phi^{2}$. We close the system and obtain (writing equations modulo $\Sigma$ ),

$$
\begin{aligned}
\phi^{1} & \wedge\left(\phi_{2}-\omega_{2}\right)+\phi_{3}^{1} \wedge \phi_{2}^{3}-\omega_{3}^{1} \wedge \omega_{2}^{3} \equiv 0, \\
\phi^{2} & \wedge\left(\phi_{1}-\omega_{1}\right)+\phi_{3}^{2} \wedge \phi_{1}^{3}-\omega_{3}^{2} \wedge \omega_{1}^{3} \equiv 0, \\
2 \phi^{1} \wedge\left(\phi_{1}-\omega_{1}\right)+\phi^{2} & \wedge\left(\phi_{2}-\omega_{2}\right)+\phi_{3}^{1} \wedge \phi_{1}^{3}-\omega_{3}^{1} \wedge \omega_{1}^{3} \equiv 0, \\
\phi^{1} \wedge\left(\phi_{1}-\omega_{1}\right)+2 \phi^{2} & \wedge\left(\phi_{2}-\omega_{2}\right)+\phi_{3}^{2} \wedge \phi_{2}^{3}-\omega_{3}^{2} \wedge \omega_{2}^{3} \equiv 0,
\end{aligned}
$$

i.e., the quadratic equations are

$$
\begin{aligned}
\phi^{1} \wedge \omega_{2}+\omega_{3}^{1} \wedge\left(y \phi^{1}+z \phi^{2}\right) & \equiv\left(a_{22}+a_{31}^{1} a_{22}^{3}-a_{32}^{1} a_{21}^{3}\right) \phi^{1} \wedge \phi^{2}, \\
\phi^{2} \wedge \omega_{1}+\omega_{3}^{2} \wedge\left(x \phi^{1}+y \phi^{2}\right) & \equiv\left(-a_{11}+a_{31}^{2} a_{12}^{3}-a_{32}^{2} a_{11}^{3}\right) \phi^{1} \wedge \phi^{2},
\end{aligned}
$$

$2 \phi^{1} \wedge \omega_{1}+\phi^{2} \wedge \omega_{2}+\omega_{3}^{1} \wedge\left(x \phi^{1}+y \phi^{2}\right)=\left(2 a_{12}-a_{21}+a_{31}^{1} a_{12}^{3}-a_{32}^{1} a_{11}^{3}\right) \phi^{1} \wedge \phi^{2}$, $\phi^{1} \wedge \omega_{1}+2 \phi^{2} \wedge \omega_{2}+\omega_{3}^{2} \wedge\left(y \phi^{1}+z \phi^{2}\right)=\left(a_{12}-2 a_{21}+a_{31}^{2} a_{21}^{3}-a_{32}^{2} a_{21}^{3}\right) \phi^{1} \wedge \phi^{2}$.

We compute the reduced polar matrix w.r.t. unknowns $\omega_{1}, \omega_{2}, \omega_{3}^{1}$ and $\omega_{3}^{2}$.

$$
\left|\begin{array}{cccc}
0 & -\phi^{1} & y \phi^{1}+z \phi^{2} & 0 \\
-\phi^{2} & 0 & 0 & x \phi^{1}+y \phi^{2} \\
-2 \phi^{1} & -\phi^{2} & x \phi^{1}+y \phi^{2} & 0 \\
-\phi^{1} & -2 \phi^{2} & 0 & y \phi^{1}+z \phi^{2}
\end{array}\right| .
$$

The determinant, a symmetric 4-tensor, is

$$
\left[x\left(\phi^{1}\right)^{2}+2 y \phi^{1} \cdot \phi^{2}+z\left(\phi^{2}\right)^{2}\right]^{2} \neq 0 .
$$

Hence, the reduced polar matrix has rank 4, which is the same as the number of independent quadratic equations. By Cartan's involutivity criteria, it follows that the system $\Sigma$ is in involution. That is to say, there exists $\bar{u}: S \rightarrow \bar{L}_{1}$ such that

$$
\left.\bar{u}^{*}\left(\omega^{i}, \Omega_{j}^{i}\right)\right|_{\bar{L}_{1}}=\left.u_{\eta}^{*}\left(\Omega^{i}, \Omega_{j}^{i}\right)\right|_{L_{1}} .
$$

From Lemma 3.7 there exist maps $\bar{\eta}: S \rightarrow N$ and $\bar{q}: S \rightarrow \bar{L}_{1} / N$ so that $\bar{u}=i_{\bar{\eta}} \circ \bar{q}$. By Lemma 4.3 this means $f$ and $\bar{f}$ are projectively equivalent to each other w.r.t. $(\eta, \bar{\eta})$. 
We now consider hypersurfaces in $\mathbf{R} P^{n}$. Let $f, \bar{f}: S^{n-1} \rightarrow \mathbf{R} P^{n}$ be hypersurfaces in $\mathbf{R} P^{n}$ locally given as graphs $\left(x^{i}\right) \mapsto\left(x^{1}, x^{2}, \ldots, x^{n-1}, f(x)\right)$ and $\left(x^{i}\right) \mapsto$ $\left(x^{1}, x^{2}, \ldots, x^{n-1}, \bar{f}(x)\right)$, where $x=\left(x^{1}, x^{2}, \ldots, x^{n-1}\right)$. Note that our index convention becomes $i \leqslant i, j, k, \cdots \leqslant p=n-1$, and $1 \leqslant \alpha, \beta, \gamma, \cdots \leqslant n$. We also let $L_{1}$ and $\bar{L}_{1}$ be bundles of first order moving frames of $f$ and $\bar{f}$, respectively. We have $u_{n}=i_{\eta} \circ q_{0}: S \rightarrow L_{1}$, where $q_{0}$ is the zero section of $L_{1} / N \rightarrow S$, and $\eta: S \rightarrow N$ is a smooth map defining a normal vector field. Also let $\left.u_{\eta}^{*}\left(\Omega^{\alpha}, \Omega_{\beta}^{\alpha}, \Omega_{\beta}\right)\right|_{L_{1}}=\left(\phi^{\alpha}, \phi_{\beta}^{\alpha}, \phi_{\beta}\right)$. Then $\phi^{n}=0$ and $\phi^{1}, \phi^{2}, \ldots, \phi^{n-1}$ form a coframe field on $S$. Note that $\left(\phi_{\beta}^{\alpha}, \phi_{\beta}\right) \in$ $\operatorname{span}\left\{\phi^{1}, \phi^{2}, \ldots, \phi^{n-1}\right\}$. Write $\left.\left(\Omega^{\alpha}, \Omega_{\beta}^{\alpha}, \Omega_{\beta}\right)\right|_{L_{1}}=\left(\omega^{\alpha}, \omega_{\beta}^{\alpha}, \omega_{\beta}\right)$, and recall that $\omega_{j}^{n}=$ $A_{j k} \omega^{k}$ for $1 \leqslant j \leqslant n-1$. From now on we will assume $\operatorname{rank}\left(A_{j k}\right)$ is maximal.

We consider the exterior system

$$
\Sigma:\left\{\phi^{i}=\omega^{i}, \phi_{j}^{i}=\omega_{j}^{i}, 1 \leqslant i, j \leqslant n-1\right\}
$$

on $S \times \bar{L}_{1}$ with designated independent variables $\phi^{1}, \phi^{2}, \ldots, \phi^{n-1}$. Note that a solution (id, $\bar{u}$ ): $S \rightarrow S \times \bar{L}_{1}$ gives a map $\bar{\eta}: S \rightarrow N$ by a decomposition $\bar{u}=i_{\bar{\eta}} \circ \bar{q}$, where $\bar{q}: S \rightarrow \bar{L}_{1} / N$, a section, and $f$ and $\bar{f}$ are projectively equivalent to each other w.r.t. $(\eta, \bar{\eta})$.

We close the system $\Sigma$ and, writing the quadratic equations modulo the system and the terms $\left(\phi^{i} \wedge \phi^{j}\right)$, obtain

$$
d \Sigma:\left\{\omega_{\eta}^{i} \wedge \omega_{j}^{n}+\omega^{i} \wedge \omega_{j}-\delta_{j}^{i} \omega_{k} \wedge \omega^{k} \equiv 0,1 \leqslant i, j \leqslant n-1\right\} .
$$

Observe that we have $(n-1)^{2}$ equations and $2(n-1)$ unknowns $\left(\omega_{n}^{i}\right)$ and $\left(\omega_{j}\right)$.

The system is in normal form, i.e., equation in $d \Sigma$ contain no quadratic terms in unknowns. (Of course, the advantages of such a system are that the variety of admissible integral elements is irreducible and the question of involutivity reduces to that of linear algebra.)

Cartan's involutivity criterion says that the system is in involution if and only if

$$
s_{1}^{\prime}+2 s_{2}^{\prime}+\cdots+(n-2) s_{n-2}^{\prime}+(n-1) \sigma_{n-1}=\operatorname{dim} V_{n-1},
$$

where $s_{1}^{\prime}, \ldots, s_{n-2}^{\prime}$ are reduced characters,

$$
\sigma_{n-1}=1+\operatorname{dim}\left(S \times \bar{L}_{1}\right)-\left(n^{2}+s_{1}^{\prime}+\cdots+s_{n-1}^{\prime}\right),
$$

and $V_{n-1}$ is the variety of admissible $(n-1)$-dimensional integral elements in $G_{n-1}\left(S \times \bar{L}_{1}\right)$, the Grassmann bundle of $(n-1)$-planes over $S \times \bar{L}_{1}$.

We compute the above numbers for $n=3$ and 4 .

(i) $n=3$. In this case we already know the equality in (4.21) must hold (Theorem 4.14). Indeed, computations show $s_{1}^{\prime}=4, \sigma_{2}=2$, and $\operatorname{dim} V_{2}=8$, giving $s_{1}^{\prime}+2 \sigma_{2}=$ $8=\operatorname{dim} V_{2}$.

(ii) $n=4$. Computations reveal $s_{1}^{\prime}=6, s_{2}^{\prime}=0, \sigma_{3}=2$ and $\operatorname{dim} V_{3} \leqslant 9$, so $s_{1}^{\prime}+2 s_{2}^{\prime}$ $+3 \sigma_{3}=12>\operatorname{dim} V_{3}$. Hence the system (4.19) is not involutive in this case.

The above discussion indicates that the problem of extrinsic projective equivalence in general is complicated. More specifically, in contrast to the $\operatorname{dim} S=2$ case, the collection of equivalence classes of induced projective structures on a hypersurface of dimension greater than two depends both on the imbedding and the choice of a normal vector field. 
5. Imbeddings of abstract projective structures. In this section we consider the problem of imbedding a given abstract projective structure. To be more precise, given an abstract projective structure $P$ over a $p$-dimensional manifold $S$, we ask if there is an integer $n \geqslant p$ and $f: S \rightarrow \mathbf{R} P^{n}$ so that $P$ is equivalent to an induced projective structure $\left(L_{1} / N, \eta\right)$ for some $\eta: S \rightarrow N$.

Let $P$ be an abstract projective structure over an (abstract) manifold $S$ of dimension $p$. Let $\left(\theta^{i}, \theta_{j}^{i}\right)=\left.\Theta\right|_{p}$ be the cannonical form of $Q(S)$ restricted to $P$. We also let $s: S \rightarrow P$ be a section and write $s^{*}\left(\theta^{i}, \theta_{j}^{i}\right)=\left(\phi^{i}, \phi_{j}^{i}\right)$. Consider the exterior system

$$
\Sigma:\left\{\phi^{i}=\Omega^{i}, \phi_{j}^{i}=\Omega_{j}^{i}, \Omega^{a}=0 ; 1 \leqslant i, j \leqslant p, p+1 \leqslant a \leqslant n\right\}
$$

on $S \times \operatorname{PGL}(n+1 ; \mathbf{R})$ (recall $\Omega=\left(\Omega^{\alpha}, \Omega_{\beta}^{\alpha}, \Omega_{\beta}\right)$ is the Maurer-Cartan form of $\operatorname{PGL}(n+1 ; \mathbf{R}))$ with specified independent variables $\phi^{1}, \phi^{2}, \ldots, \phi^{p}$. Suppose we have a solution of the form $(s, u): S \rightarrow P \times \operatorname{PGL}(n+1 ; \mathbf{R})$. Define $f: S \rightarrow \mathbf{R} P^{n}$ by $f \equiv \pi \circ u$. Then we observe that $u$ is actually a first order moving frame field along $f$ since $u^{*}\left(\Omega^{a}\right) \equiv 0$ by hypothesis. As before, we can decompose $u=i_{\eta} \circ q: S \rightarrow L_{1} / N$ $\rightarrow L_{1}$. Now it is easy to see that $P$ is realized as (i.e., equivalent to) $\left(L_{1} / N, \eta\right)$ :

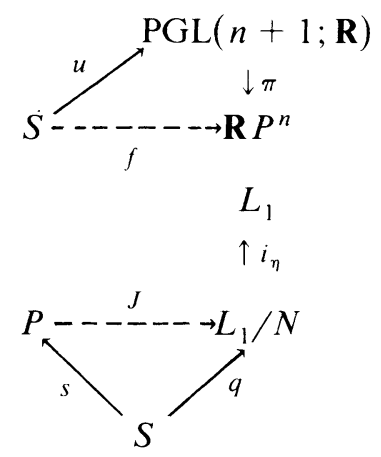

The system (5.1) should be compared with that obtained in [6]. Suffice it to say that we have a smaller number of equations, and the minimal dimension $n(p)$ required to imbed abstract projective structures over a $p$-dimensional manifold is considerably smaller than that obtained by Chern. This is to be expected as projective connections are more "rigid" than projective structures. (Here, the author is thankful to the referee for pointing out the analogous situation in conformal geometry.)

We prove the following special case.

THEOREM 5.2. Let $f: S \rightarrow \mathbf{R} P^{3}$ be any real analytic nonplanar surface and $P$ any abstract projective structure over $S$. Then there exists $\eta: S \rightarrow N$ so that $P$ is equivalent to $\left(L_{1} / N, \eta\right)$.

Proof. Let $\left(\phi^{i}, \phi_{j}^{i}\right)$ be as in the above discussion, i.e., $\left(\phi^{i}, \phi_{j}^{i}\right)=s^{*}\left(\theta^{i}, \theta_{j}^{i}\right)$ :

$$
s \int^{P} \downarrow
$$


Also let $\omega^{i}=\left.\omega^{i}\right|_{L_{1}}$ and $\omega_{j}^{i}=\left.\Omega_{j}^{i}\right|_{L_{1}}$. We consider the exterior system

$$
\left\{\phi^{i}=\omega^{i}, \phi_{j}^{i}=\omega_{j}^{i}, 1 \leqslant i, j \leqslant 2\right\}
$$

on $S \times L_{1}$ with independent variables $\phi^{1}$ and $\phi^{2}$. This system has the same polar matrix as (4.15), hence it is in involution. The rest follows easily.

\section{REFERENCES}

1. E. Cartan, Sur la déformation projective des surfaces, Ann. Sci. École Norm. Sup. 37 (1920), 259-356.

2. Théorie des groupes finis et continues et la géométrie différentielle traitées par la méthode du repère mobile, Gauthier-Villars, Paris, 1937.

3. ___ Les systèmes différentiels extérieurs et leurs applications géométriques, Hermann, Paris, 1945.

4. __ Sur la connexion projective des surfaces, Oeuvres Complètes, Vol. I, 1955, pp. 915-918.

5. S. Chern, The geometry of G-structures, Bull. Amer. Math. Soc. 72 (1966), 167-219.

6. Sur la possibilité de plonger un espace à connexion projective donné dans un espace projectif, Selected Papers, Springer-Verlag, New York, 1978, pp. 35-44.

7. C. Ehresmann, Introduction à la théorie des structures infinitésimales et des pseudo-groupes de Lie, Colloque de Geométrie Différentiel, Strasbourg, 1953.

8. G. Jensen, Higher order contact of submanifolds of homogeneous spaces, Lecture Notes in Math., vol. 610, Springer-Verlag, Berlin, 1977.

9. Deformation of submanifolds of homogeneous space, J. Differential Geom. 16 (1981), 213-246.

10. S. Kobayashi, Transformation groups in differential geometry, Ergebnisse Math. Grenzgeb. Bd. 70, Springer-Verlag, Berlin, 1972.

11. S. Kobayashi and T. Nagano, On projective connections, J. Math. Mech. 13 (1964), 215-236.

12. S. Kobayashi and K. Nomizu, Foundations of differential geometry, Vols. I. II, Interscience, New York, 1963.

13. S. Sternberg, Lectures on differential geometry, Prentice-Hall, Englewood Cliffs, N. J., 1964.

Department of Mathematics, Washington University, St. Louis, Missouri 63130

Department of Mathematics, Arkansas State University, State University, Arkansas 72467 